\title{
Application of Botulinum Toxin Injection in Symmetric and Asymmetric Gummy Smile Cases
}

${ }^{1}$ Nilsun Bagis, ${ }^{2}$ Rahme Barbaros, ${ }^{3}$ Hasan Yildiz

\begin{abstract}
Gummy and asymmetric smile was affecting patients' selfconfidence and life standards. Immediate, nonsurgical botox applications delivered painless and rapid solution to the patients. It has been shown that botulinum toxin type A (BTX-A) injection, which is used in correcting excessive gingival display caused by hyperactive upper lip elevator muscles, is quite effective in gummy and asymmetric smile cases. The patients were reasonably satisfied with the outcomes of the treatment. In spite of the transient effect of BTX-A, even after 6 months of treatment, it was revealed that the gummy and asymmetric smile was within normal limits and did not fully return. For this reason, depending on the needs of the patient, BTX-A injection can be used as an alternative procedure for faster and minimally invasive treatment of gummy and asymmetric smile cases.

The purpose of this study is to evaluate the efficacy of conservative treatments like BTX injections in asymmetric and gummy smile cases.
\end{abstract}

Keywords: Asymmetric smile, Botulinum toxin, Gummy smile, Nonsurgical smile design.

How to cite this article: Bagis N, Barbaros R, Yildiz H. Application of Botulinum Toxin Injection in Symmetric and Asymmetric Gummy Smile Cases. Int J Experiment Dent Sci 2018;7(1):39-42.

Source of support: Nil

Conflict of interest: None

\section{INTRODUCTION}

Among all facial expressions, smile is regarded as the most charming and complex expression. When human history is examined, it is said that one of the most effective means by which people express their feelings is smile ${ }^{1}$; $2 \mathrm{~mm}$ or more of gingival display upon smiling is described as gummy smile. Extreme gingival display upon smiling may be esthetically uncomfortable for patients. There are many factors that play a role in gummy smile cases, and one of them is hyperfunctional lip elevator muscles. ${ }^{2}$ It is known that the extreme gingival display on smiling is

\footnotetext{
${ }^{1}$ Associate Professor, ${ }^{2,3}$ Research Assistant

${ }^{1-3}$ Department of Periodontology, Faculty of Dentistry, Ankara University, Ankara, Turkey
}

Corresponding Author: Nilsun Bagis, Associate Professor Department of Periodontology, Faculty of Dentistry, Ankara University, Ankara, Turkey, Phone: +903122965640 , e-mail: nilsunbagis@yahoo.com caused by the difference between the lower edge of the upper lip and the upper margin of the incisor teeth, which is considered as the most embarrassing esthetic problem in humans. There are several methods to treat gummy and asymmetric smile cases, depending on the condition of the patient, such as orthodontic surgeries like LeFort I osteotomy, orthodontic treatment, crown lengthening, gingivoplasty, and BTX injections. ${ }^{3}$

The use of BTX in cosmetic purposes, over the past two decades, has quickly become one of the most effective treatment options for improving esthetic facial conditions. For a long time, BTX has been used for the treatment of wrinkles in the upper third of the face area as the most preferred treatment option. In addition to this, it has recently been used in other areas, including in the treatment involving the middle and lower thirds of the face and the neck to prevent and correct the changes caused by muscle contraction, such as gummy smile. ${ }^{1}$

Since the 1970s, BTX has been used extensively in the treatment of different problems associated with pain and extreme muscle contraction. Botulinum toxin is produced from an anaerobic bacterium called Clostridium botulinum. The BTX has eight different serotypes, the most effective and most commonly used serotype being BTX-A. Purified BTX-A, a stable, sterile, vacuum-dried powder diluted with a preservative-free saline solution, occurs as a consequence of $C$. botulinum fermentation. ${ }^{4}$ The toxin induces a transient state of muscle paralysis as a result of inhibiting presynaptic acetylcholine release at the neuromuscular junction. This chemical denervation effect has been effectively applied in esthetic plastic surgery where low doses can be used to reduce the effects of hyperkinetic facial muscles. ${ }^{5,6}$

The purpose of this report is to show the use of BTX-A for the treatment of gummy and asymmetric smile as a nonsurgical, fast, painless, and noninvasive method.

\section{MATERIALS AND METHODS}

\section{Case 1}

A 28-year-old female patient came to Ankara University Faculty of Dentistry, Department of Periodontology with the complaint of extreme gingival display during smiling which affected her self-confidence and psychological state. According to anamnesis obtained, the patient had 


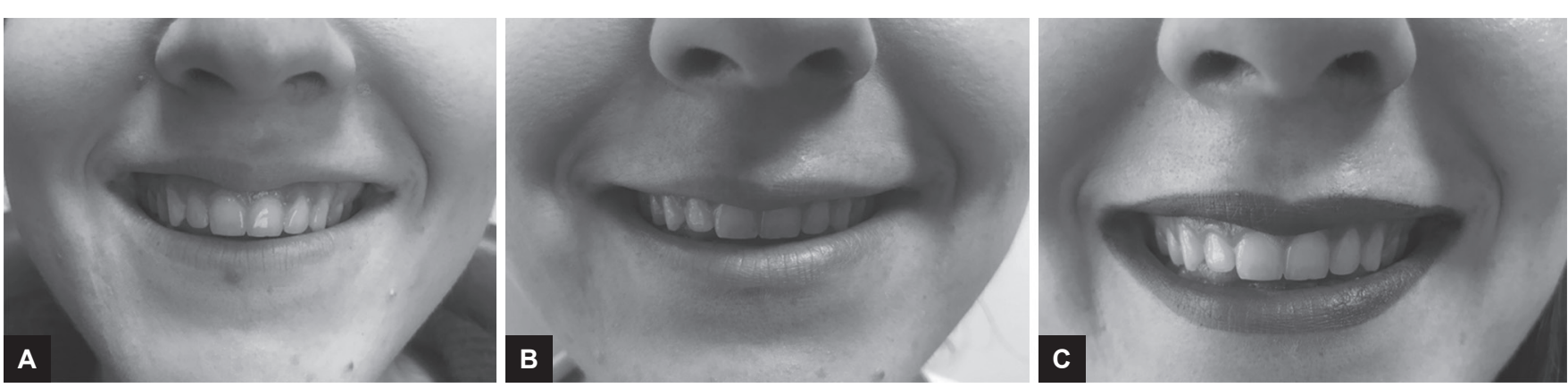

Figs 1A to C: (A) Before BTX application. Botulinum toxin was injected to the left and right side. (B) After 15 days of treatment. (C) 6th month follow-up

not reported any systemic diseases or drug use. Oral examination revealed that the skeletal jaw relationship of the patient was normal and the etiology of gummy smile was due to hypermobile upper lip.

Since no prosthetic restoration was planned for the patient, crown lengthening, one of the treatment options, was not preferred due to risk of sensitivity development after surgery. The second option, lip repositioning technique, was denied by the patient due to fear of surgical procedure. Botulinum toxin injection, which is a noninvasive, nonsurgical procedure that does not need intraoral application, was more preferable by the patient.

A 2.5 unit BTX-A (BOTOX ${ }^{\circledR}$ onabotulinumtoxinA, Allergan, Dublin, Ireland) injection was planned to the left and right side of musculus levator labii superioris muscles. Fifteen days later, the patient was recalled to the clinic. Since the patient's satisfaction was high as a result of the controls performed at 1,3,6 months, the application was not repeated (Fig. 1).

\section{Case 2}

A 23-year-old female patient referred to our clinic reported dissatisfaction with the excessive gingival exposition and asymmetry during her smile. According to anamnesis obtained, she was systemically and dentally healthy with unremarkable use of any drugs. In the examination performed, it was observed that the left upper lip movement was higher than the right and musculus levator labii superioris was more active. A single-sided 2.5 unit BTX-A injection was planned. On the 15th day, the asymmetry of the upper lip was less, but since it did not completely disappear, 2.5 unit injections were applied to the same point again. Patient satisfaction was significantly high at 1-month follow-up (Fig. 2).

\section{DISCUSSION}

Surgical techniques, such as orthognathic surgery, modified lip repositioning, or crown lengthening can be used for gummy smile treatment. In recent times, BTX application has been used as an alternative to other treatment options since it has a minimally invasive treatment approach. There are various options of surgeries for gummy smile if the etiologic factor is skeletal and the patient approves to have a surgery. Rubinstein and Kostianovsky ${ }^{7}$ introduced a method in which the elliptical part of the gingiva and buccal mucosa is excised and the wound areas were approximated and sutured in the study they performed. Litton and Fournier ${ }^{8}$ investigated a treatment method that separates the muscles from the bony structures with detachment and as a result of this brings the upper lip down. Silva et $\mathrm{al}^{9}$ applied modified lip repositioning technique to the patients with gummy smile. According to the results of these studies, the patients were satisfied, but there are various complications and risks due to surgical techniques. Surgical methods can often cause unwanted side effects, such as
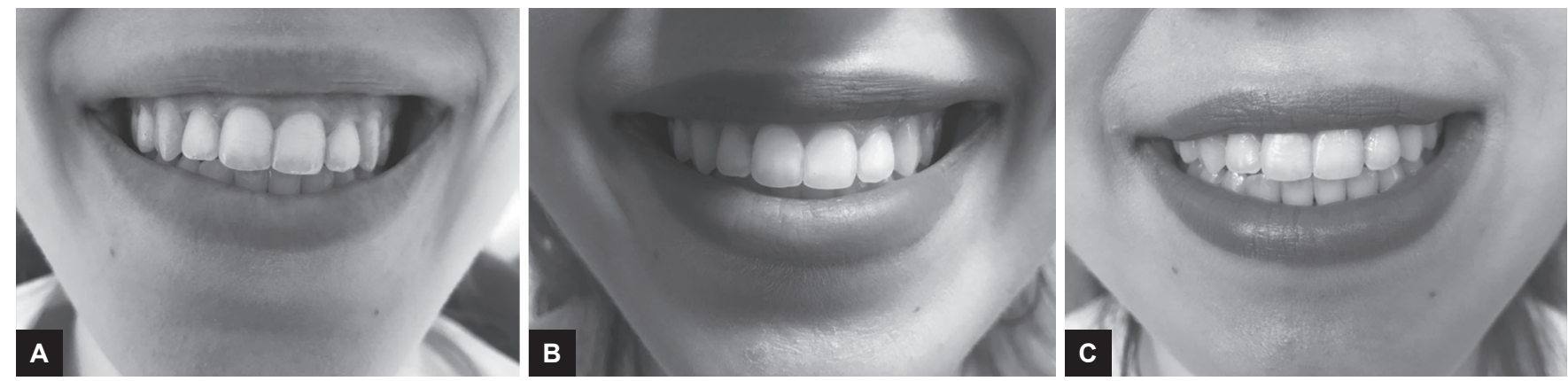

Figs 2A to C: (A) Before BTX application. A single-sided BTX was injected to the left side. (B) After 15 days of treatment. Botulinum toxin was injected again. (C) 1st month follow-up 
relapse and scar formation. Because of all side effects, noninvasive BTX treatments are considered to be more comfortable, easier, and painless methods for patients. Due to all these reasons, BTX applications in dentistry are becoming more popular day by day.

The literature on BTX application frequency and dose has been researched. The BTX should be injected with small, carefully titrated doses to limit excessive contraction of the upper lip muscles. Hwang et $\mathrm{al}^{10}$ recommended an injection point for BTX and called it as Yonsei point. This point is mainly located at the center of the triangle formed by the levator labii superioris, levator labii superioris alaeque nasi, and zygomaticus minor. According to this protocol, a 3-unit dose is recommended for each injection site. Also the frequency of BTX-A application (more than 12 weeks) is another point that should be careful since repeated applications can result in the formation of neutralizing antibodies against toxins, which can lead to disappointing and undesired results. The effect of BTX-A lasts for 6 months except for more serious cases ( 3 months). The application interval is between 4 and 8 months, and this time should be respected because there is accumulation of antibodies that reduces the effect of BTX-A. ${ }^{11,13}$

The injection dose varies among studies. In a study by Nayyar et $\mathrm{al}^{12}{ }^{12}$ they treated five patients with extreme gingival exposure emerging from hyperfunctional upper lip elevator muscles with BTX injections. It was noted that 2.5 units BTX per muscle was applied to the patients bilaterally into the levator labii superioris, levator labii superioris alaeque nasi, levator labii superioris, and zygomaticus minor muscles. According to the results of this study, the duration of the effect varied from 3 to 6 months and no side effects were reported. However, the observed improvement after botox administration is transient and should be repeated every 6 months to 1 year. Considering this study, in case 1, 2.5 units of BTX injection was applied from one point both on the right and left side. We observed that the efficacy of BTX decreased at the 6th month control but did not completely disappear.

Brum et $\mathrm{al}^{13}$ reported that the application area was the nose wing sideways at two points (Yonsei Point). Both sides had a 2-unit Botox application and no additional application was needed. The maximum effect of improvement was recognized after 15 days, but clinical results were obtained for 6 months.

Although it is a simple and safe procedure, BTX injection may be linked with some side effects, such as infection, pain in the injection site, edema, ptosis or upper lip tension, and smile asymmetry. It is crucial for the dentist to analyze the dosage, technical certainty, and the area of injection. ${ }^{14,15}$ The clinical effects of BTX applications come out within 2 to 10 days after injection and the highest visible effect occurs 14 days after injection. ${ }^{11,13}$

There are some contraindications in the use of BTX. It is recommended not to use in the case of pregnancy; hypersensitivity to BTX itself or lactose and albumin; muscular and neurodegenerative diseases, such as myasthenia gravis, Eaton-Lambert syndrome myasthenia, amyotrophic lateral sclerosis, and the simultaneous use of aminoglycoside antibiotics that can enhance the toxin effect. In addition, there may be drug interactions of BTX-A with calcium channel blockers, cyclosporines and cholinesterase inhibitors. All these situations should be evaluated carefully before BTX application. ${ }^{11,12,14}$

In spite of its reversibility via neuromuscular complex and some adverse effects, BTX-A injections appear to be the least invasive treatment option for gummy and asymmetric smile cases.

\section{CONCLUSION}

It has been shown that BTX-A injection, which is used in correcting excessive gingival display caused by hyperactive upper lip elevator muscles, is quite effective in gummy and asymmetric smile cases. The patients were reasonably satisfied with the outcomes of the treatment. In spite of the transient effect of botox, even after 6 months of treatment, it was revealed that the gummy and asymmetric smile was within normal limits and did not fully return. For this reason, depending on the needs of the patient, this treatment approach may be used as an alternative procedure for faster and minimally invasive treatment of gummy and asymmetric smile cases.

\section{REFERENCES}

1. Patel D, Mehta F, Trivedi R, Thakkar S, Suthar J. Botulinum toxin and gummy smile-a review. IOSR J Dent Med Sci 2013 Jan-Feb;4(1):1-5.

2. Suber JS, Dinh TP, Prince MD, Smith PD. OnabotulinumtoxinA for the treatment of a "gummy smile". Aesthet Surg J 2014 Mar;34(3):432-437.

3. Al-Fouzan AF, Mokeem LS, Al-Saqat RT, Alfalah MA, Alharbi MA, Al-Samary AE. Botulinum toxin for the treatment of gummy smile. J Contemp Dent Pract 2017 Jun;18(6):474-478.

4. Ananthanarayana, R.; Jayaram Paniker, CK. Textbook of microbiology. 7th ed. Hyderabad: Orient Longman; 2005.

5. Rohrich RJ, Janis JE, Fagien S, Stuzin JM. The cosmetic use of botulinum toxin. Plast Reconstr Surg 2003 Oct;112(5 Suppl):177S-188S.

6. Carruthers JD, Glogau RG, Blitzer A; Facial Aesthetics Consensus Group Faculty. Advances in facial rejuvenation: botulinum toxin type a, hyaluronic acid dermal fillers, and combination therapies-consensus recommendations. Plast Reconstr Surg 2008 May;121(5 Suppl):5S-30S.

7. Rubinstein AM, Kostianovsky AS. Cosmetic surgery for the malformation of the laugh: original technique. Prensa Med Argent 1973;60:952. 
8. Litton C, Fournier P. Simple surgical correction of the gummy smile. Plast Reconstr Surg 1979 Mar;63(3):372-373.

9. Silva CO, Ribeiro-Junior NV, Campos TV, Rodrigues JG, Tatakis DN. Excessive gingival display: treatment by a modified lip repositioning technique. J Clin Periodontol 2013 Mar;40(3):260-265.

10. Hwang WS, Hur MS, Hu KS, Song WC, Koh KS, Baik HS, Kim ST, Kim HJ, Lee KJ. Surface anatomy of the lip elevator muscles for the treatment of gummy smile using botulinum toxin. Angle Orthod 2009 Jan;79(1):70-77.

11. Borodic G. Immunologic resistance after repeated botulinum toxin type a injections for facial rhytides. Ophthalmic Plast Reconstr Surg 2006 May-Jun;22(3):239-240.
12. Nayyar P, Kumar P, Nayyar PV, Singh A. Botox: broadening the horizon of dentistry. J Clin Diagn Res 2014 Dec;8(12): ZE25-ZE29.

13. Brum CR, Coleta RD, Lunardi N, Boeck EM, Pizzol KE. The use of botulinum toxin as a way of treatment for gingival smile: case report. J Res Dent 2015 Sep-Oct;3(4): 798-807.

14. Jaspers GW, Pijpe J, Jansma J. The use of botulinum toxin type A in cosmetic facial procedures. Int J Oral Maxillofac Surg 2011 Feb;40(2):127-133.

15. Mazzuco R, Hexsel D. Gummy smile and botulinum toxin: a new approach based on the gingival exposure area. J Am Acad Dermatol 2010 Dec;63(6):1042-1051. 